\title{
Recuperación del patrimonio mediante prácticas artísticas contemporáneas
}

\author{
María González Fernández \\ Universidad Politécnica de Valencia
}

RESUMEN:

Muchos de los habitantes de los espacios en los que se desarrollan las actuales prácticas artísticas contemporáneas, se vieron abandonados ante la falta de perspectivas económicas, culturales y por supuesto, educativas en el medio rural español a partir de los años sesenta del siglo XX. En la actualidad, proyectos culturales como La Harinera, en Pedro Muñoz, Castilla La Mancha; la FCAYC, Fundación Cerezales Antonino y Cynia, en Cerezales del Condado, León; Paraisu Rural en Villaviciosa, Asturias; o Scarpia en El Carpio, Córdoba, unifican el arte, la arquitectura, la sociología, la antropología cultural, la música, los estudios del entorno y les prestan atención a las necesidades sociales para reconfigurar la cultura como identidad individual y social en el medio rural.

PALABRAS CLAVE:

Arte contemporáneo, recuperación del patrimonio, comunidad rural, social, sostenibilidad.

\section{ABSTRACT:}

Many of the inhabitants of the areas in which current contemporary art practices developed were abandoned due to the lack of economic, cultural and educational course of the Spanish countryside from the sixties of the twentieth century perspectives. Today cultural projects as The Harinera in Pedro Muñoz, Castilla La Mancha; the FCAYC Foundation Cerezales Antonino and Cynia in Cerezales, Lion; Paraisu Rural in Valdedios, Asturias; or Scarpia in El Carpio, Cordoba, Andalusia unify art, architecture, sociology, cultural anthropology, music, environmental studies and provide them with attention to the needs reconfiguring social culture as individual and social identity at rural areas.

KEYWORDS:

Contemporary art, heritage restoration, rural community, social, sustainability. 
1. El arte rural en el contexto de la institución en España

En la actualidad, existen varios calificativos para denominar la práctica artística en el medio rural; ya sea land art, arte en la tierra, que establece un diálogo entre los materiales propios del lenguaje plástico y el medio ambiente, designado por artistas en la década de los años sesenta; neorruralismo, como lo llama Joan Nogué i Font ${ }^{1}$, en 1988, o iniciativas independientes, también denominadas alternativas autogestionadas, término con la que lo define el historiador Frank Mintz como "una gestión autónoma y surge de la necesidad en las comunidades de provincias rusas hacia sus vidas sostenibles en medio del abandono de los poderes feudales y la aristocracia"2. La mejor forma de explicar este modo de creación será diciendo que se trata de un tipo de corriente artística que se articula en torno a varios conceptos que se pueden transmitir en el ámbito rural. Es decir, la búsqueda para dar forma activa al proceso creativo en el medio rural y al trabajo intelectual basado en una metodología de trabajo integradora y participativa, procurando la activación de procesos relacionales y cooperativos entre todas las personas que forman parte de un modo activo de estas corrientes culturales contemporáneas desarrolladas en el ámbito rural.

El acercamiento del arte al territorio rural en España, se ve imbricado con los movimientos migratorios de la ciudad al campo surgidos en España a partir de mayo del 68, sencillo eco de los realizados en Estados Unidos y Europa en la década de los 60; únicamente cambia de dimensión para adaptarse al contexto cultural, económico, político y social propio de los 60, y por extensión, del último tercio del S. XX.

En España se formaron las primeras comunidades migratorias como Arco Iris, en Areyns de Muns, Barcelona; Casa María, en Herrerías, Santander; Ilícitis, en Elche, etc. Éstas, como las europeas, tienen el denominador común de su migración en la reivindicación de la "contracultura americana" y los movimientos generados en Europa, y en especial en Francia en mayo

NOGUÉ I FONT, Joan, El fenómeno neorrural, Barcelona, pp 145- 147. En url http://www.magrama. gob.es/ministerio/pags/biblioteca/revistas/pdf_ays/ a047_06.pdf.

2 LADAGA, Reinaldo, Estética de la emergencia; la formación de otra cultura de las artes, Buenos Aires, Ed., Adriana Hidalgo, 2006. del 68. En la actualidad existen corrientes ligadas a un territorio que se identifican con el arte y la naturaleza en el arte contemporáneo, estas se han expresado a través de intervenciones en el medio natural, y se han dado propuestas artísticas relacionadas con el arte ecológico ${ }^{3}$ como denominador común, cuyos artistas son herederos del Land Art, pero a diferencia de ellos se oponen a la sobredimensión de la escala y a la transformación evidente recuperando para el arte una poética de la preservación del entorno.

Los movimientos americanos y europeos del Land Ar, Earth Works, Art in Nature, Arte Povera, etc coinciden con el nacimiento de la conciencia ecológica y de los movimientos sociales de defensa del medioambiente. Las intervenciones del Land Art presentan un carácter agresivo hacia el paisaje, para los artistas "son la colonización conceptual y física"4, en cambio en Europa las prácticas artísticas no suponen ningún daño para el ecosistema, encontrando un equilibrio entre la obra y el entorno natural preservando la naturaleza.

En España museos como el Museo Vostell Malpartida, fundado en 1979 en Cáceres, e instituciones privadas como el CDAN, Centro de Arte y naturaleza, inaugurado en 1999, en Huesca, gestionado por la Fundación Beulas, son ejemplos pioneros de las sedes artísticas del Land Art en las instituciones españolas. Estos aprecian el intercambio de cultura con lo perteneciente o relativo al medio rural para lograr resultados que respondían a las necesidades del siglo XX por la ausencia de políticas culturales en el mismo.

\section{El potencial de las fundaciones a principios del S.XXI}

En 2008 nace la Fundación Cerezales Antonino y Cynia, FCAYC, en la localidad de Cerezales del Condado, León, de donde es originario su fundador Antonino Fernández, empresario y emigrante local. Su sede está en las antiguas escuelas rehabilitadas del pueblo, de cuya rehabilitación se encargó la propia fundación. La institución, de carácter privado, está gestionada por Rosa Yagüez y Alfredo Puente quienes, junto a su equipo, se ocupan del desarrollo del

ALBELDA, José ; SABORIT, José, La construcción de la naturaleza, Generalitat Valenciana, 1997.

4 ALBELDA, José ; SABORIT, José; Op. Cit., p. 94. 


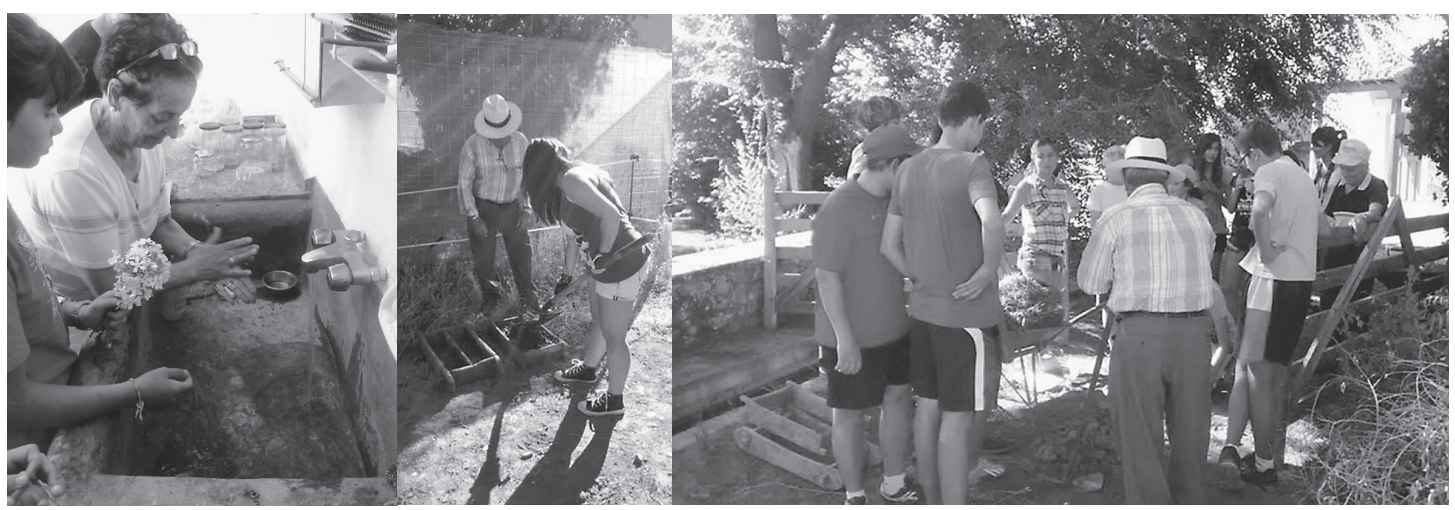

Fig 1. De izquierda a derecha: Taller de creación de jabón; Taller de realización de adobe; Taller de construcción de ladrillos de adobe para la Azada de Oro, Residencia artística del colectivo Paraisu Rural, Cerezales del Condado, León, 2014. Fuente: Archivo personal del colectivo Paraisu Rural.

territorio y la transferencia de conocimiento a la sociedad mediante la producción cultural y la etnoeducación, haciendo uso de las tecnologías libres o creative commons.

El uso de estas tecnologías en los proyectos que se trabajan en la fundación, son nexos comunes en los artistas que han trabajado para la misma, y su uso facilita la colaboración de un modo abierto y participativo a personas de cualquier edad que habitan en la localidad durante todo el año o solo en los meses estivales, pero que intervienen con su información en los diversos proyectos que allí se realizan. De este modo, la fundación unifica el trabajo entre archivistas, agricultores, amas de casa, artistas, investigadores, jóvenes, jubilados y docentes, unificando conocimientos mediante la transmisión oral, recetas gastronómicas compiladas, folclore, trabajos comunes o filandones, y todo esto se recogen en bibliotecas digitales a las que existe un libre acceso posterior para todos los públicos. Muestra de esto es el proyecto iniciado en base al taller del artista en residencia Daniel García Andujar ${ }^{5}$, junto con Abelardo Gil-Fournier, cuyo trabajo critica el uso de las tecnologías y las condiciones socio- políticas que definen su uso y su producción, y crean redes de colaboración en torno a los distintos

GARCÍA ANDUJAR, Daniel, (Almohadí) 1966. «Miembro histórico de irational.org, referente internacional del arte en la red, Fundador de Technologies To The People y director de proyectos en internet como art. net.dortmun, e-barcelona.org. Ha dirigido numerosos talleres para artistas y colectivos sociales en diferentes países» Cfr. Autobiografía de Daniel García Andújar para la web de Daniel García, en url: http://www.danielandujar.org/cv-espanol/ temas y las convierte en plataformas de investigación.

La Fundación está dirigida a todos los públicos, sus actividades transversales promueven la música, el medioambiente, la sociología y la economía; dichas actividades se realizan a lo largo de todo el año dando lugar a exposiciones temporales, talleres y residencias artísticas. Las residencias son compaginadas con talleres realizados por los artistas para niños y jóvenes en período estival y se amplían a todo el año unificadas a su programación educativa en grupos de trabajo reducidos para todas las edades. FCAYC patrocina diversas líneas de trabajo de artistas que trabajan con el ecosistema rural, desde un punto de vista etnológico como el proyecto Herbarium de Lorena Loza$\mathrm{no}^{6}$, o $\mathrm{El}$ Cazador, proyecto fotográfico que estudia la supervivencia del ser humano en territorios internacionales de Álvaro Laíz ${ }^{7}$. En la actualidad, la fundación trabaja en la con-

\footnotetext{
LOZANO, Lorena, (Gijón) 1975 «La Bióloga Lorena Lozano desarrolla el proyecto Herbarium en la Fundación Cerezales Antonino y Cinia de León. Es miembro fundacional y coordinador del proyecto Ecolab, en la Laboral Centro de Arte y Creación Industrial de Gijón» Cfr. Autobiografía de Lorena Lozano para la web de Econodos, en url: http://lorenalozano.net/

7 LAÍZ, Álvaro, (León) 1981. "Su trabajo se ha desarrollado fundamentalmente en África, Asia y Sudamérica colaborando con fundaciones y ONG's como Cruz Roja, Médicos sin fronteras y World Vision... Utiliza la fotografía como herramienta con que dotar de voz a la sociedad civil en zonas de post-conflicto, explorando el entorno, las costumbres y las tradiciones de las minorías en riesgo de exclusión.» Cfr. biografía de ÁlvaroLaíz para la web de ANHUA org, en url: http:// www.an-hua.org/es/sobre/alvaro-laiz
} 


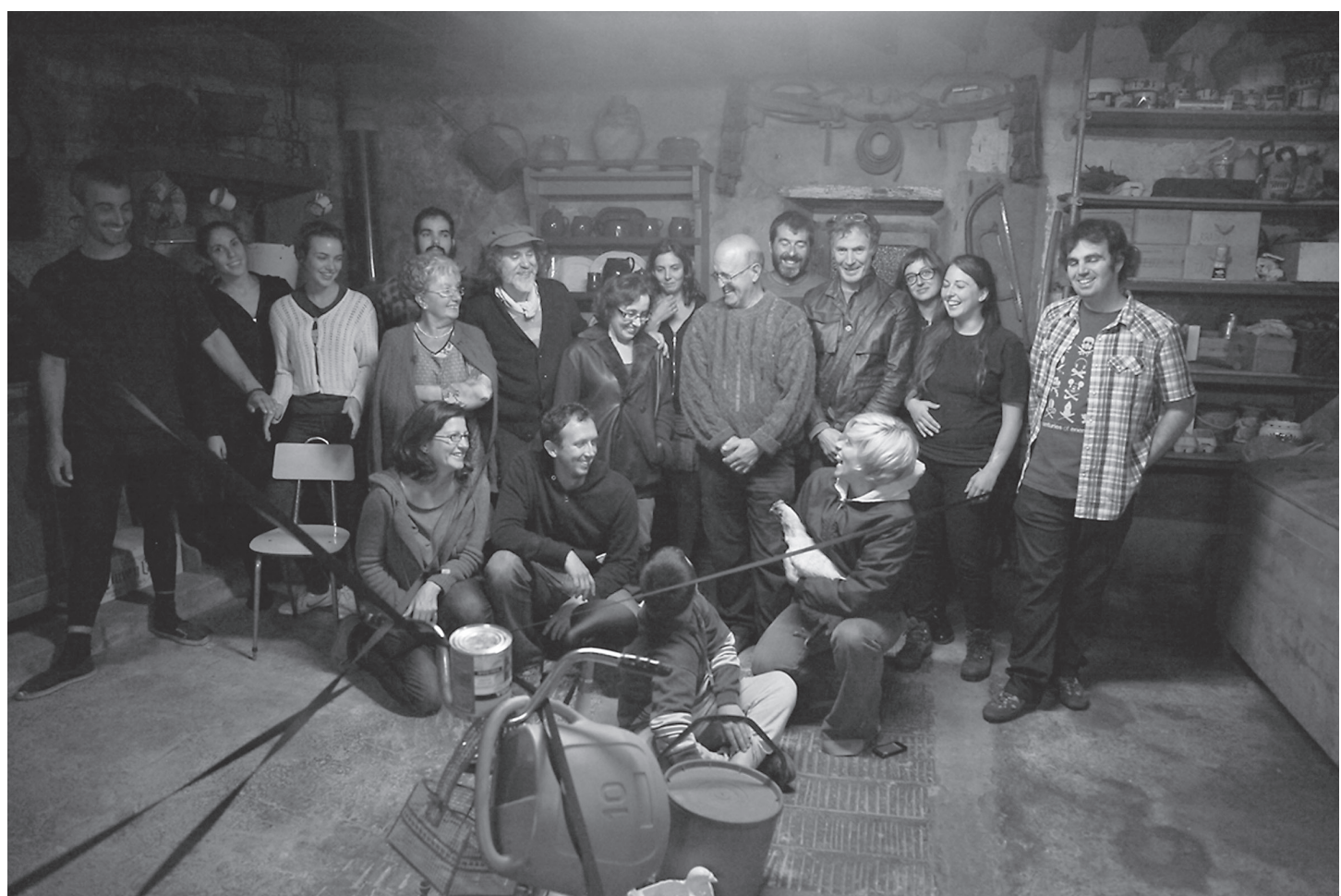

Fig. 2. Taller de arte de acción realizado por Alba Soto, Performance, Vallinaoscura, en Villaviciosa, Asturias, Primera edición de Paraisu Rural, 2012. Fuente: Emanuele Giusto, fotógrafo.

tinuación de Territorio Archivo ${ }^{8}$, otro proyecto cuya primera parte ha sido recopilado en una publicación. Este proyecto originado desde la fundación compila información sobre su territorio rural, las condiciones físicas y culturales entre las comarcas de Cerezales y Curueño en León. Desde 2013 la Fundación Sánchez Ruipérez en Peñaranda de Bracamonte, Salamanca, colabora también con FCAYC ampliando la investigación realizada para Territorio Archivo al ámbito rural de Salamanca. Esta continuación se lleva a cabo por los habitantes de la propia comunidad salmantina, en torno a la tradición oral, la poesía y la música.

Coetánea a la Fundación Cerezales, surge, en 2010, Campo Adentro, un proyecto de territorios, geopolítica, cultura e identidad en el contexto de las relaciones entre lo urbano y lo rural. Este está dirigido por Fernando García Mory y depende de una subvención por los fondos Feder y El Museo Reina Sofía entre otros. Este proyecto es pionero en el modo de repensar el arte en el medio rural en la primera década del S. XXI en España, puesto que ofrece la oportunidad de analizar las actuales per-

8 DOMINGUEZ, Chus; BLASCO, Jorge, Territorio Archivo, León, 2014 cepciones y representaciones de la vida rural en diversas localidades españolas y ver cómo influye en la construcción de su identidad cultural. Su objetivo es proporcionar una interpretación de la vida rural que ponga de relieve las amenazas y oportunidades que existen en el campo español desde el punto de vista de la cultura contemporánea. Campo Adentro poseyó, en sus principios, residencias para artistas en poblaciones rurales a lo largo de la Península Ibérica y ofrecía al artista la posibilidad de desarrollar proyectos artísticos durante su estancia o presentarlos ejecutados aunque no tuvieran relación con las áreas de interés del programa. La residencia constaba de inmersión artística plena en el mundo rural y las obras de arte se mostraban de un modo público junto con una presentación y una publicación final. Las instalaciones eran en diversas localidades de España: Coria en Cáceres, Tierra de Barros en Badajoz, la aldea de Valladares en Pontevedra, Farrera en Pallars de Sobirá, Lérida, Puebla de la Sierra en Madrid, Vallehermoso en la isla de La Gomera, en las Islas Canarias entre otras. En 2013 las condiciones del proyecto varían y pasa a desarrollarse dentro de las Naves de la Música del Centro de creación contemporánea Matadero Madrid, compuesto este por siete ins- 
tituciones que engloban todas las disciplinas artísticas. Allí, Campo Adentro realiza talleres, exposiciones y mesas debate en torno al arte rural, el arte público, la historia, la política y los nuevos usos del bien común; en la actualidad, se realiza una publicación que compila la información sobre la experiencia, el método seguido y su desarrollo en el ámbito rural.

Un caso similar y pionero en Castilla La Mancha se lleva a cabo a principios del año 2013, desde la localidad de Pedro Muñóz, en Ciudad Real, esta acoge el primer centro de arte contemporáneo y desarrollo de la cultura artística en el medio rural: La Harinera. Su presidente, Santiago Arroyo, trabaja desde hace años en la gestión cultural, y junto a su equipo ha llevado a cabo este proyecto que vincula el patrimonio industrial, la innovación cultural y la creación contemporánea en una antigua fábrica de harinas y pan. El proyecto comienza con la rehabilitación de la fábrica de harinas del siglo XIX, la restauración de una de sus naves y la realización de un albergue cercano en el que realizar intercambios artísticos, como miembros de la red de residencias artísticas internacionales Res artis.

La Harinera pone en práctica una línea de trabajo a través del fomento de la creatividad de manera sostenible y, como las anteriores fundaciones o proyectos citados, se desarrolla en las tradiciones locales integrando el arte y la innovación para contribuir a la transformación social en el medio rural. Este proyecto emergente ha desarrollado una gran actividad desde febrero de 2014: la puesta en marcha del propio centro junto con la apertura de la sala principal; la primera residencia de artistas emergentes con “Arenillas y Fuentesal”, Julia Fuentesal y Pablo Muñóz de Arenillas, que intervinieron temporalmente el espacio interpretando el carácter de la localidad en la que se ubica La Harinera, (la dialéctica entre lugar o presencia como lo hicieron los ya consagrados Antoni Muntadas, Francesc Torres, Nam Yum Paik o Mucha); se inauguró la primera exposición individual de la reinterpretación del Greco a través del 3D en una muestra pictórica; se realizaron conciertos, la apertura de un albergue para las residencias artísticas, y el acercamiento de todo ello a los habitantes de Pedro Muñoz y las localidades cercanas. En la actualidad se trabaja en convertir el patrimonio industrial de la Harinera en un museo social.
3. El potencial de las iniciativas independientes

Del mismo modo que los museos y las fundaciones privadas muestran en la actualidad un acercamiento del estado del arte rural en el patrimonio de la península ibérica, rehabilitando arquitectura industrial y social para sus sedes y realizando investigaciones de recuperación de la cultura local, conviven con los anteriores las iniciativas independientes, también denominadas alternativas o autogestionadas. En ellas el arte abandona las instituciones y los espacios cerrados y se muestra mediante proyectos colectivos en espacios independientes, para visibilizar otros proyectos que a pesar de no depender de las instituciones son parte significante de la actual transformación cultural en proyectos comunitarios o sociales tanto en la ciudad como en el ámbito rural. Estos proyectos parten de la premisa de estar gestionados por artistas y activistas culturales. Las iniciativas independientes se caracterizan por su heterogeneidad organizativa (organizaciones horizontales de producción, sin patronos o al menos con la involucración y participación de los componentes que conforman la organización más o menos igualitaria), por la diversidad de sus propuestas estéticas y por realizar un trabajo muy cercano a los movimientos sociales. Su vinculación con el movimiento asociativo es, de hecho, innegable debido a su planteamiento colectivo y voluntad de transformación social. Su principal interés no se focaliza en producción de la obra artística, sino en el desarrollo y proceso en sí, y en la intervención espontánea de los asistentes, que han dejado de ser sujetos pasivos y alcanzan la expresión artística a través de la representación o intervención colectiva. Por este motivo, las iniciativas independientes se desarrollan en lugares públicos, o que fueron públicos en su momento, y en la actualidad se han convertido en sedes culturales, movilizando, acercando y haciendo partícipes del arte y la cultura a los habitantes de las localidades rurales, irrumpiendo su cotidianidad, lo que nos hace repensar los paradigmas institucionales, como los museos y las instituciones privadas.

En el asociacionismo, según Melucci ${ }^{9}$ la identidad colectiva es el primer elemento de unión, es como "una definición compartida e

\footnotetext{
9 MELUCCI, A (1994), “¿Qué hay de nuevo en los movimientos sociales?”, p.120, en E. Laraña, Los nuevos
} 


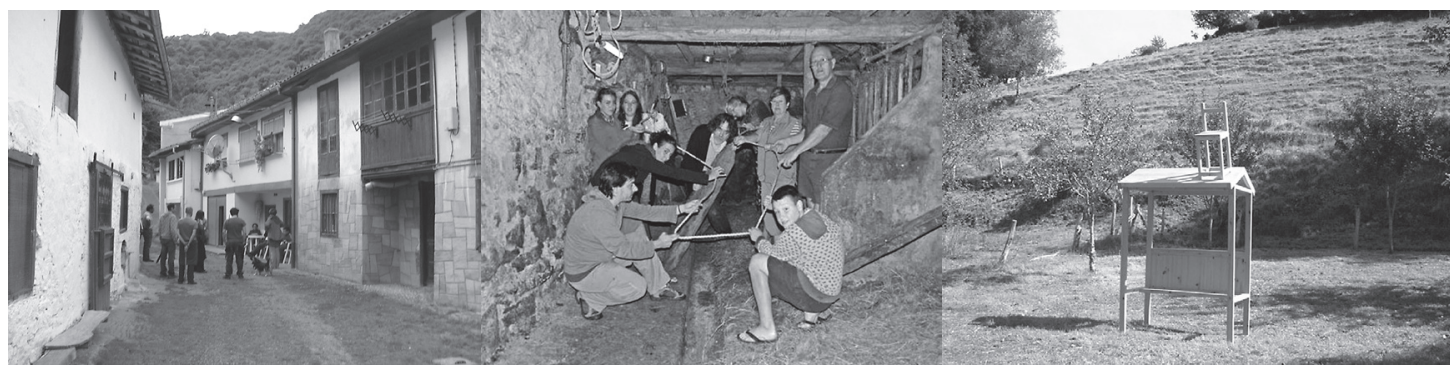

Fig. 3. De izquierda a derecha: Localización de espacios para las intervenciones, y trabajos colaborativos previos a la realización del proyecto Paraisu Rural, barrio de Villarica, en Villaviciosa, 2011. Fuente: Archivo personal del colectivo Paraisu Rural. Retrato de Anxel Nava, miembros de Nodal Mode, estudio de arquitectura, Diógenes Ferrándiz, Elisa Cuesta y Estela Asensio, alumnos de la Universidad Antonio Nebrija de Madrid, y Alberto Berros con Julita Meana, habitantes de Vallinaoscura, en la primera edición de Paraisu Rural, 2012. Fuente Periódico La Nueva España, sección central. Silla sobre el tejado, en lugar del pájaro por Adolfo Manzano, Instalación, barrio de Santi, en Villaviciosa, Asturias, Primera Edición Paraisu Rural, 2012. Fuente: Archivo personal del colectivo Paraisu Rural.

interactiva producida por varios individuos- o por grupos a nivel más complejo- que está relacionada con las orientaciones de la acción y con el campo de oportunidades y constricciones en las que ésta tiene lugar". En este sentido se desarrolla Scarpia en El Carpio, localidad rural en la provincia de Córdoba, un proyecto artístico dirigido por Miguel Ángel Moreno Carretero. Nace en 2012 gracias a la colaboración de este y del historiador del arte Antonio R. Sánchez Gavilán, quienes ante el desconocimiento del arte público en la región de Andalucía crean un proyecto de intervenciones artísticas y talleres en el medio rural. Ambos, junto a miembros del ayuntamiento del Carpio, artistas vinculados a la enseñanza y un fotógrafo relacionado con dicho pueblo, forman un equipo que trata de relacionar a jóvenes creadores andaluces con artistas de prestigio. Su finalidad es desarrollar los aspectos formales y teóricos, y visualizar así desde un pueblo las diversas formas de expresión visual contemporánea. Todo ello se unifica con la problemática de aceptación e integración de los propios habitantes del pueblo en el proyecto, dada la carencia cultural que existía en la localidad sobre este tipo de intervenciones artísticas en espacios públicos. Ha de tenerse en cuenta que estas se encuentran alejadas de las grandes urbes como pueden ser Madrid o Barcelona, donde existe un mayor conocimiento del arte contemporáneo. Por ello Scarpia desarrolla también formación no reglada, y pretende unificar las distancias de las enseñanzas básicas en educación que facilitan la comprensión y adhesión de cualquier persona en un proyecto de tal envergadura.

movimientos sociales: de la ideología a la identidad, Madrid, Ed. Alianza, 1999, pp 119- 150.
En un principio sus destinatarios eran exclusivamente personas de la localidad, sin embargo, en la actualidad, Scarpia se ha configurado como empresa de gestión cultural. A lo largo del año se realizan talleres culturales, cursos para niños, jóvenes y adultos, se organizan ciclos y jornadas formativas y se presenta proyectos de características similares de otras localidades cercanas, como es el caso de La Fresnedilla, proyecto agroartístico en Obejo, Córdoba.

La formación de esta iniciativa como empresa de gestión cultural se debe a las diversas posturas internas en el equipo, y a las necesidades territoriales que el proyecto ha tenido como su ampliación en colaboraciones con otras residencias artísticas como La Fragua, en el convento de Santa Clara de Belalcazar en los Pedroches, al norte de Córdoba, o el proyecto internacional Artifariti, que desarrolla unas jornadas de arte en el desierto del Sahara promovidas por el Ministerio de Cultura de la República Árabe Saharaui Democrática (RASD) y la Asociación de Amistad con el pueblo Saharaui de Sevilla.

Durante doce ediciones en Scarpia se han sucedido múltiples participaciones y colaboraciones con artistas y colectivos nacionales e internacionales como Boamistura, colectivo de Madrid que trabaja las intervenciones pictóricas en arquitectura; Basurama, colectivo que trabaja a partir de materiales reciclados; arquitectos como Santiago Cirujeda ${ }^{10}$ y sus Arquitecturas Colectivas; y artistas audiovisuales como Eugenio Ampudia. El proyecto Scarpia no deja de ser una realidad más que nos muestra el arte ${ }_{10}$ CIRUGEDA, Santiago, Arquitecturas colectivas, Sevilla,
Ed, Vibok Works, 2010. 
tal y como cita Miguel Ángel Moreno «una forma de creer en el trabajo, y que el arte es una forma de vida y no una forma de ganarla» ${ }^{11}$.

\section{Paraisu Rural, una práctica artística en el entorno rural asturiano}

La preocupación por el estado del arte contemporáneo también desarrolló una concepción crítica y participativa entorno al ámbito rural y a las políticas participativas en la comunidad autónoma asturiana. A finales de 2011, miembros de diversos colectivos asturianos junto con artistas, historiadores, docentes y arquitectos se unifican en el Colectivo Paraíso de Oviedo, y emprenden un proyecto que se abre a las especificidades, a la emergencia y visibilización de comunidades o espacios que trabajan con la producción y la distribución de conocimiento a través de proyectos colectivos artísticos en los contextos locales y experiencias culturales en el ámbito rural.

De este modo se crea Paraisu Rural, un proyecto colectivo de artistas, arquitectos, creativos e investigadores cuyo objetivo fundamental es, además de la organización y el desarrollo del conocimiento artístico, su aplicación en la colaboración y toma de decisiones con las personas que viven en el medio rural. La iniciativa colabora con la localidad en la que se desarrolla ayudando en el rediseño de la agenda pública del ámbito rural y local, dialogando con las políticas rurales, medioambientales, culturales, educativas y tecnológicas desarrolladas en la actualidad, que influyen de manera efectiva en la configuración de lo local y en las condiciones de vida del ámbito rural y su entorno más cercano, como expresión de aquello por construir, aquello que está sucediendo o por suceder.

Este proyecto se inicia en el Valle de Boiges, en Vallinoscura, localidad cercana a Valdedios, perteneciente al Ayuntamiento de Villaviciosa, aldea cuyos hórreos están catalogados como patrimonio artístico debido a las tallas y pinturas, de los siglos XIV y XV, que en sus liños y puertas se encuentran. Paraisu Rural impulsa

"11 MORENO, Miguel Ángel (1980),” Scarpia XIII reflexionará sobre el paisaje a través del concepto e "retrato"”, Salamanca, 2014. En url http://www.theartboulevard.org/es/lab-esp/informaci\%C3\%B3n-0C3\%BAtil/ post/1550/el-proyecto-scarpia-o-c\%C3\%B3mo-introducir-el-arte-p\%C3\%BAblico-en-el-0C3\%A1 mbit la participación directa de personas a una experiencia a través del arte con el mundo rural. Una experiencia que atiende a intervenciones escultóricas, dibujos, instalaciones, light painting (pintura con luz), acciones, performance, talleres artísticos (estampación xilográfica, dibujo al natural, expresión corporal), música y la realización de talleres de prácticas tradicionales relacionadas con oficios tan diversos como la apicultura, la restauración de tambores, la cestería, la gastronómica local y las sextaferias, o trabajos comunales, para el mantenimiento del entorno, entendiendo la cultura como una noción que abarca las capacidades creativas y sociales. Dichas capacidades comprenden no solo la producción artística, sino también la acción social, el pensamiento crítico y la difusión de ideas, obras y procedimientos que buscan expandir y recuperar zonas rurales o potenciarlas a través del arte.

Paraisu Rural se dirige a toda la población activa y los habitantes del Valle de Boiges y sus alrededores. Grabadores, escultores, grafiteros, dibujantes, gaiteros, performers, pintores, alfareros, maestros de las antiguas escuelas taller, agricultores, ganaderos, hilanderas, biólogos, $\mathrm{y}$ personas residentes en el municipio se han dedicado durante tres años a mostrar y transmitir sus conocimientos sobre las disciplinas artísticas tradicionales y contemporáneas, y los diversos oficios existentes. La creación de este proyecto, en primer lugar, nace y crece a través de lazos informales con los vecinos, las instituciones locales, los peregrinos del camino de Santiago, hasta llegar a las instituciones públicas y privadas, desde lo más inmediato a lo más alejado. La propia elección del espacio corresponde con la casa de uno de los componentes del colectivo, de esta forma se cede una casa familiar desde los primeros encuentros hasta sus relaciones con familiares, amigos, y vecinos del pueblo para buscar implicación en el proyecto desde el inicio. De un modo paralelo surge la búsqueda de artesanos locales o del entorno, a quienes se les pide apoyo y búsqueda de puntos de encuentro para la creación de talleres o sinergias diversas durante los encuentros que se han llevado a cabo de una forma bianual desde el año 2012. El colectivo cultural como núcleo de apoyo se une para trabajar en el proceso de preparación, difusión y posterior montaje e instalación de obras, y por último con la Fundación Cardín como institución local más sensible a actividades artísticas, que colaboró 


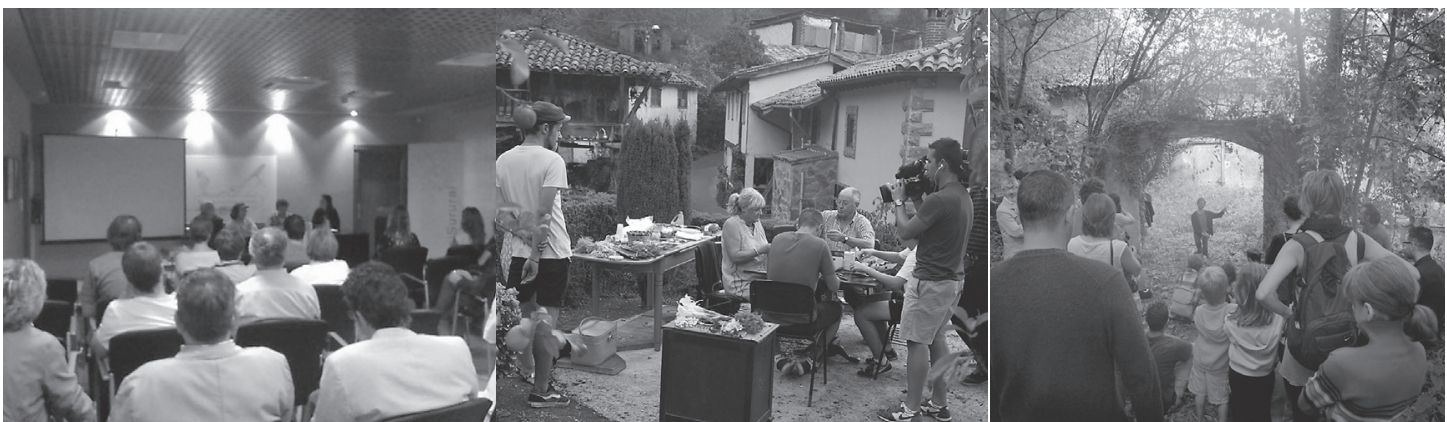

Fig. 4. De izquierda a derecha: Ponencias de inauguración y presentación del proyecto realizadas por Anxel Nava, Myrem González y otros miembros del colectivo, Fundación José Cardín, en Villaviciosa, Primera Edición de Paraisu Rural, 2012. Fuente: Archivo personal de colectivo Paraisu Rural. Taller de creación de esculturas efímeras por Sergio Santurio, Taller de arte contemporáneo, Vallinaoscura, en Villaviciosa, Segunda Edición Paraisu Rural, 2014. Fuente: Emanuele Giusto, fotógrafo. Interpretación de Sierra Musical, Compañia Cía\&Pandemonium, Concierto de inauguración, Valdedios, en Villaviciosa, Asturias, Segunda Edición de Paraisu Rural, 2014. Fuente: Archivo personal del colectivo Paraisu Rural.

aportando apoyo técnico y difusión al núcleo de población más habitado de la zona, Villaviciosa. Así el primer paso en Vallinaoscura, parte de visitas previas al espacio durante varios meses, interrelacionando los miembros de los colectivos, los habitantes de la localidad rural y algunos de los artistas nacionales e internacionales. Estos encuentros constituyeron la realización de una convocatoria pública de proyectos artísticos para desarrollar en los espacios públicos y privados del pueblo y el entorno de Valdediós. Entornos abiertos para el desarrollo de la actividad artística de los interesados, y la realización de una programación de talleres por artesanos locales y artistas. El proyecto se abrió también a la vertiente educativa, y se planteó una práctica artística a la Universidad Antonio Nebrija de Madrid, ofreciendo el espacio como residencia artística a estudiantes de los grados de bellas artes, arquitectura y diseño de interiores.

El proyecto explora ciertos valores del tejido social y unifica la producción cultural y artística, desde la participación y el territorio en el que se desarrolla. La producción aúna el arte y su contexto, los interrelaciona en las diversas disciplinas para que se retroalimenten y se vuelve transversal en los estados del arte público, el arte privado, el ámbito social y el contexto rural. Se valora el trabajo en el medio, el respeto al entorno, la convivencia y las relaciones que se dan junto con las artes plásticas y escénicas, las nuevas tecnologías, los talleres, la artesanía y sus lenguajes, la difusión y la formación cultural en el cuidado mutuo.

Situados en el contexto, el proyecto Paraisu Rural se ha realizado en dos ediciones en el año 2012 y 2014 en el mes de septiembre. En la primera edición, durante siete días, participaron treinta artistas, nacionales e internacionales, relacionados con las artes plásticas, escénicas y musicales, tres alumnos en residencia de la Universidad Antonio Nebrija de Madrid, de los grados de BBAA, diseño de interiores y arquitectura, seis artesanos y catorce agricultores y ganaderos/as, que convivieron en cuatro aldeas del municipio de Villaviciosa: La Campa, Villarica, Vallinaoscura y La Viña. La segunda edición, realizada durante cuatro días, contó con la participación de diez artistas plásticos, cinco músicos, un alumno en residencia de la Universidad Antonio Nebrija de Madrid, seis agricultores/as y ganaderos/as en Vallinaoscura y Valdedios, y se amplió con la primera residencia de cuatro artistas nacionales. Escultores como Adolfo Manzano y Pablo Maojo, performers como Alba Soto, Anxel Nava, Nera S. Lorences, artistas emergentes como María Castellanos, Sergio Santurio, o el colectivo Children Of Darklight, y artistas audiovisuales como Cesar Naves, Jaime Rodríguez junto con el colectivo Mind Revolution, han sido algunos de los artistas colaboradores en el proyecto, obteniendo así un público de más de cien visitas en cada edición.

Paraisu Rural se considera un factor de desarrollo rural a través del arte y crea un espacio de desarrollo artístico en el medio rural en el que se realice. Se supone de necesidad tanto a nivel social como económico y cultural. La elaboración del mismo ha conllevado que se rehabiliten las antiguas escuelas de Puelles como local cultural, antigua sede de la Asociación Amigos del Paisaje de Valdedios, donde se realizan diversas actividades relacionadas con la música a lo largo de todo el año. Dadas las características del proyecto, se requiere la 


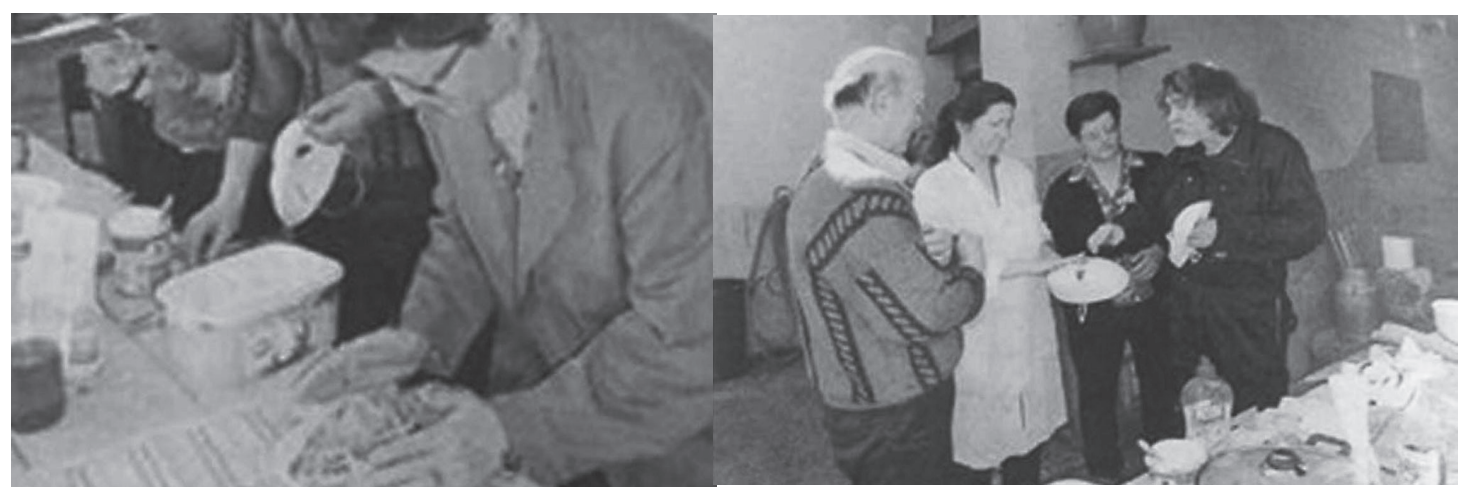

Fig 5: Taller de máscaras, por Anxel Nava y miembros de la familia Berros. Trabajo de intercambio de conocimientos para la recuperación del carnaval tradicional en febrero, en Vallinaoscura, 2014. Fuente: Periódico La Nueva España, sección central.

colaboración y participación de los habitantes del lugar, para generar reciprocidad y revalorar el patrimonio artístico de la localidad en la que se desarrolla. Compartir el arte en sus disciplinas más diversas en los espacios públicos y privados del medio rural, cuyo fin es transmitir las tradiciones orales y las prácticas que se realizan en el entorno inmediato, sin olvidarnos de los organismos oficiales y entidades privadas como el Ayto. de Villaviciosa, la Fundación José Cardín, El Centro Capistrano, y el actual recuperado Ateneo Obrero de Villaviciosa, entre otros.

En poco más de un año, el proyecto se ha convertido en una organización de referencia más allá del territorio local. Las personas que desarrollan la iniciativa participan en sus actividades, la utilizan como lugar de encuentro, la visitan o solicitan al colectivo de su colaboración para pensar en nuevos proyectos e intervenciones. Alberto Berros, habitante de Vallinaoscura, ha propuesto su casa como posible residencia artística para próximas ediciones, algunos miembros del colectivo han realizado talleres para fundaciones como muestra de su método creativo, y artistas como Anxel Nava, realizan un trabajo de investigación y restauración del Carnaval, antroxu en lengua asturiana, donde el artista recopila muestras de la tradi- ción oral en la zona, archivando y clasificando coplas y cantares, así como la restauración del attrezzo popular para dar constancia de este y sus similitudes con los retratos del pintor Evaristo Valle.

La incidencia social del proyecto no se mide solo por las apariciones en medios de comunicación, tanto reportajes en la televisión local, Rtpa, como en entrevistas y retransmisiones en directo desde RTVE o la COPE, ni en sus intervenciones en los Encuentros Internacionales de Juventud de Cabueñes, en Gijón, las ponencias en la Universidad Antonio Nebrija, de Madrid, las visitas a su web, www.paraisurural.com, o las solicitudes como residencia artística por artistas nacionales en la actualidad. Paraisu Rural fue y es objeto de estudio de tesis doctorales de la Universidad de Oviedo y la Universidad Politécnica de Valencia, sus instalaciones y sus recursos se utilizan como apoyo para propuestas sociales y culturales desde el ámbito local hasta las instituciones nacionales en las que ha colaborado. Y, lo más importante, quienes acuden, pasean, viven, participan y construyen este proyecto de arte en el medio rural, encuentran lo que hallan en instituciones museísticas o fundaciones privadas culturales pero de un modo vivencial, en relación con el entorno que lo crea y lo acoge. 
